\title{
THE TUNEL ASSAY IN THE DIAGNOSIS OF GRAFT-VERSUS-HOST DISEASE: CAVEATS FOR INTERPRETATION
}

\author{
Keith R. Jerome*, Claudio Vallan $\dagger$ and Rolf JaGgi ${ }^{\dagger}$ \\ *Department of Laboratory Medicine, University of Washington, Seattle, Washington, USA, and \\ $\dagger$ Department of Clinical Research, University of Bern, Switzerland
}

\begin{abstract}
Summary
Acute graft-versus-host disease (GVHD) is a significant cause of morbidity and mortality following bone marrow transplantation, and early detection is important to allow effective therapy. Since the presence of apoptotic keratinocytes (dyskeratotic bodies) has been suggested as a useful diagnostic criterion for GVHD, attention has focused on the use of the TUNEL assay to detect apoptosis in clinical specimens. We reviewed clinical specimens upon which TUNEL had been performed for possible artifacts that might interfere with accurate evaluation for GVHD. Several distinct types of artifact were found and could be re-created in experimental systems. Artifacts in TUNEL staining generally resulted from the lack of specificity of this reaction for apoptotic cell death. Artifacts were found resulting from inadequate fixation, over-exposure of the TUNEL reaction, and proximity to the section edge. In addition, a novel artifact, apparently resulting from DNA shearing during the sectioning process, was noted and confirmed using confocal microscopy of experimental specimens. The TUNEL assay must therefore must be interpreted with caution in the clinical setting. In our laboratory, we consider TUNEL-positive cells as apoptotic only when accompanied by apoptotic morphology. Although these criteria clearly miss some cells in the early stages of apoptosis, they provide the highest specificity for apoptotic cell death.
\end{abstract}

Key words: TUNEL, apoptosis, graft-versus-host, artifact, laser confocal scanning microscopy

Abbreviations: LCSM, laser confocal scanning microscopy; GVHD, graftversus-host disease; TUNEL, Terminal deoxynucleotidyl transferase (TdT)-mediated dUTP-biotin nick end labeling.

Accepted 20 February 2000

Acute graft-versus-host disease (GVHD) remains a significant cause of morbidity and mortality following bone marrow transplantation (BMT). The pathology of GVHD appears to be multifactorial in origin, resulting from both a direct attack upon recipient tissues by immunocompetent cells from the donor as well as derangements of immune regulatory mechanisms with release of inflammatory cytokines (the so-called "cytokine storm"). These mechanisms most likely work together to cause the pathology of GVHD, which is most pronounced in the skin, GI tract, and liver.
GVHD is treated most effectively when detected early, and in most institutions early diagnosis is based on skin biopsy. Unfortunately, the histopathological features of GVHD overlap with other skin pathology frequently seen in BMT recipients, such as drug reactions or alterations secondary to pre-transplant conditioning regimens. Among the criteria useful to distinguish GVHD from these processes, some authors have focused on the presence of "dyskeratotic bodies". We and others have shown that the presence of such bodies, together with other findings such as an activated lymphomonocytic infiltrate, supports the diagnosis of GVHD. ${ }^{1-3}$ With the recent realisation that these dyskeratotic bodies actually represent apoptotic keratinocytes, ${ }^{4,5}$ attention has focused on methods such as the TUNEL reaction (terminal deoxynucleotidyl transferase (TdT)-mediated dUTP-biotin nick end labeling) which in theory might allow more sensitive detection of apoptotic cells in the skin. However, our experience with the TUNEL assay suggests that there are a number of commonly occurring artifacts that can lead to potential misinterpretations in the evaluation of skin biopsies for GVHD. The TUNEL assay must be interpreted with caution in regard to these potential artifacts to prevent erroneous diagnosis of GVHD. In this report we illustrate artifacts that can lead to erroneous interpretation of TUNEL results, re-create these artifacts in experimental systems, and describe the appearance and causes of previously undescribed artifacts.

A large number of biochemical and other tests now exist to study the process of apoptosis in cultured cells, including assays based on morphological changes, oligonucleosomal DNA fragmentation, cell membrane phospholipid distribution, caspase activation, mitochondrial transmembrane potential and many others. However, there has been a clear need among pathologists and other scientists for methods capable of detecting apoptosis in intact tissue specimens. In 1992, Gavrieli et al. described a procedure for the detection of DNA fragmentation in situ, by end labeling of DNA strand breaks using terminal deoxyribonucleotidyl transferase $(\mathrm{TdT}){ }^{6}$ Since double-stranded DNA breaks occur during apoptosis, TdT would be expected to preferentially interact with such cells. At the broken DNA ends, TdT catalyses template-independent addition of labeled deoxyuridine triphosphates (dUTPs), which can then be detected by immunohistochemical or other means. Since the introduction of the TUNEL assay a wide variety of commercial kits have been introduced, based on this or similar technology, and there has been increased interest in the possible clinical utility of TUNEL-based testing. The TUNEL assay can be 

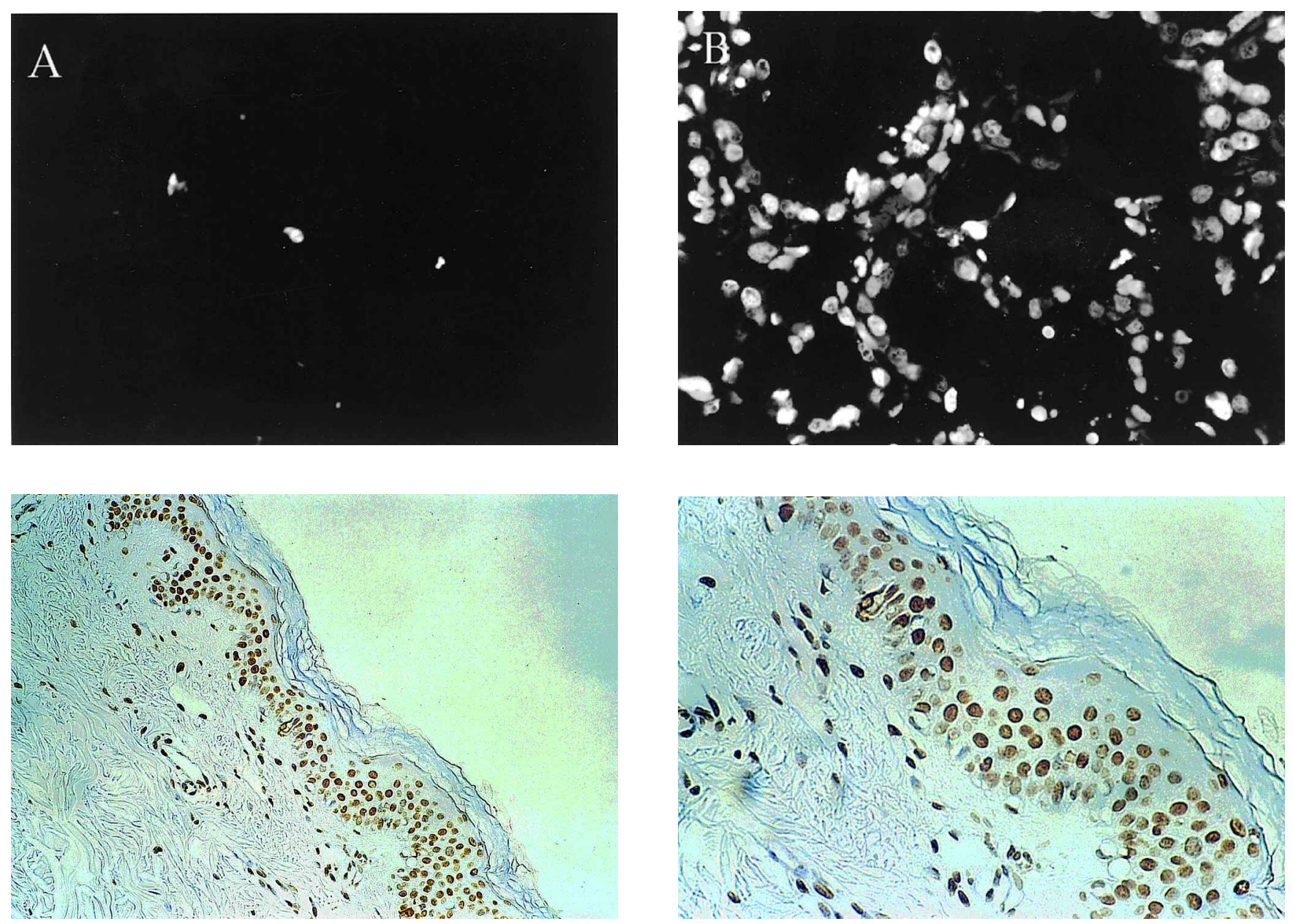

Fig. 1 Overstaining artifact in TUNEL sections. (A) Laser confocal scanning microscopy (LCSM) of fluorescent-labeled TUNEL reaction of involuting mouse mammary gland, LCSM gain set to low sensitivity. TUNEL reactions on mammary gland sections were performed as described by Gavrieli et al., ${ }^{6}$ using digoxygenin-11-dUTP and anti-digoxygenin FITC-labeled Fab fragments (Boehringer Mannheim, Mannheim, Germany). The low sensitivity in this case is analogous to a properly stained peroxidase reaction, and shows isolated nuclei with apoptotic morphology, as expected in the mammary gland model. (B) LCSM of the same area as (A) with gain set for high sensitivity. The image shows positive signal in essentially all nuclei, analogous to an overstained peroxidase specimen. (C) Clinical GVHD specimen showing overstaining artifact, with positive signal from essentially all nuclei. TUNEL staining on clinical specimens was performed using the In Situ Cell Death Detection Kit, POD (Boehringer Mannheim), according to the manufacturer's instructions. (D) Higher-power view of (C), illustrating lack of apoptotic morphology in TUNEL-positive cells.

performed in as little as $2-4 \mathrm{~h}$ after specimen fixation and rehydration, and thus is available within a clinically relevant time frame.

Unfortunately, the very mechanisms that allow the TUNEL reaction to occur also lead to, perhaps, its most serious general limitation: the TUNEL assay also stains cells which have died by non-apoptotic mechanisms. ${ }^{7,8}$ Grasl-Kraupp et al. demonstrated, using a rat liver model, that the TUNEL assay stains not only apoptotic hepatocytes, but also cells that have died via necrosis. Since cells dying by a necrotic mechanism can show extensive DNA fragmentation $^{9}$ with plentiful free DNA ends, it is not surprising that such cells can be labeled in the TUNEL reaction. In some systems, necrotic versus apoptotic TUNEL-positive cells can be differentiated by their morphological appearance. ${ }^{10}$ Although in many diagnostic situations the exact mechanism of death (apoptotic versus necrotic) may be relatively unimportant, in biopsies taken for the diagnosis of GVHD necrosis of individual keratinocytes might occur as a result of high-dose radiation treatment pre-BMT. Therefore, the presence of TUNEL positivity in such cells should not be taken as proof of apoptotic cell death.

Adequate fixation of tissue is a critical factor for the proper use of the TUNEL technique. Fixation can be especially problematic in larger tissue sections, in which significant differences in TUNEL reactivity can be seen between well-fixed areas on the periphery of the specimen, and the relatively unfixed areas in the center of the specimen. In general, poorly fixed areas will show increased signal in the TUNEL reaction. A similar increased signal is seen with extended delays before fixation. These signals do not represent true apoptosis, but rather areas of autolytic cell death occurring after removal of the tissue. Tateyama et al. have shown that the number of TUNEL-positive cells can increase two- to three-fold with a 12-h or greater delay in proper fixation of tissue. ${ }^{11}$ Fortunately, the small size of the skin biopsies used in the diagnosis of graft-versus-host disease generally ensures proper fixation throughout the tissue, as long as the specimen is placed in fixative within a reasonable time after it is obtained. In our institution, skin specimens are placed into fixative immediately after the 

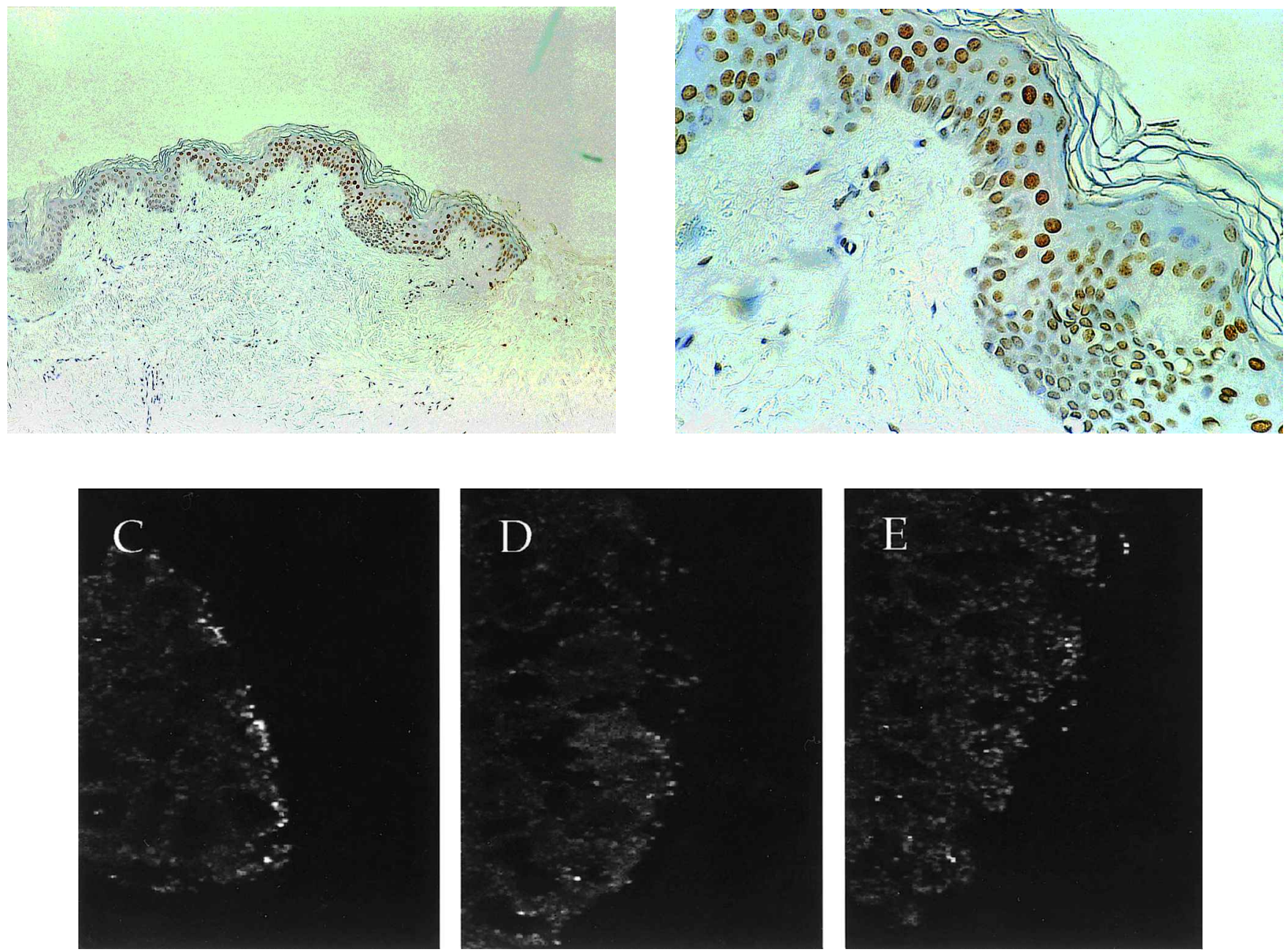

Fig. 2 Artifactual TUNEL positivity near borders of sections. (A) Clinical GVHD specimen, showing enhanced TUNEL positivity near border (right side of specimen), and lack of TUNEL staining away from border (left side). (B) Higher power view of (A), showing lack of apoptotic morphology in TUNEL-positive cells. (C-E) LCSM images of fluorescent-labeled lactating mammary gland. Despite the expectation of few or no apoptotic cells at this stage of glandular development, TUNEL-positive cells are consistently found along borders of specimen.

biopsy is taken, obviating this as a concern in subsequent TUNEL analysis.

As described earlier, the TUNEL reaction is based upon the addition of varying numbers of nucleotides to the free $3^{\prime}$ termini produced by double- or single-strand DNA breaks. Thus, the sensitivity of the reaction is highly dependent upon the exact conditions used. Each laboratory must therefore empirically determine conditions under which the TUNEL assay will reliably detect cells undergoing apoptosis as judged by the morphology of their nuclei. While it is likely that the conditions thus chosen will miss some cells in the early stages of apoptosis (before morphological changes have occurred), these conditions will ensure maximal specificity of the TUNEL reaction. DNA strand breaks are found in most cells in fixed specimens, either due to autolysis, fixation artifact, or the cutting procedure, and such breaks can be detected by greatly increasing the TUNEL reaction (overstaining) or increasing the sensitivity of detection (Fig. 1A,B). In clinical specimens, such overstained sections may show large areas in which the majority of cells show TUNEL reactivity (Fig. 1C). Despite the TUNEL reactivity, the cells show none of the morphological features of apoptosis (nuclear shrinkage, chromatin condensation or retraction from surrounding cells) (Fig. 1D). Clearly, a positive TUNEL signal obtained under such conditions would be of questionable diagnostic value.

We have also frequently seen artifactual TUNEL positivity near the borders of the skin biopsy sections we have studied (Fig. 2A). We interpret this reactivity as artifact because the incidence of TUNEL positivity in these sections is markedly higher at the edges than near the center of the section, where the number of TUNEL-positive cells is more in line with expectations. Furthermore, close examination of the cells showing TUNEL positivity in these sections reveals that very few had the characteristic morphology of apoptosis (Fig. 2B). Similar artifacts can be reproduced in experimental systems such as the involuting mouse mammary gland, in which TUNEL positivity was again highest near the borders of sections (Fig. 2C-E). TUNEL positivity at the borders was seen not only in the ductal epithelial cells, where it might be expected, but also among stromal cells, which generally show much less apoptosis in involuting tissue. The high incidence of TUNEL positivity among stromal cells in this system supports the artifactual nature of the staining near borders. For these reasons, in diagnostic specimens we generally only evaluate areas of 

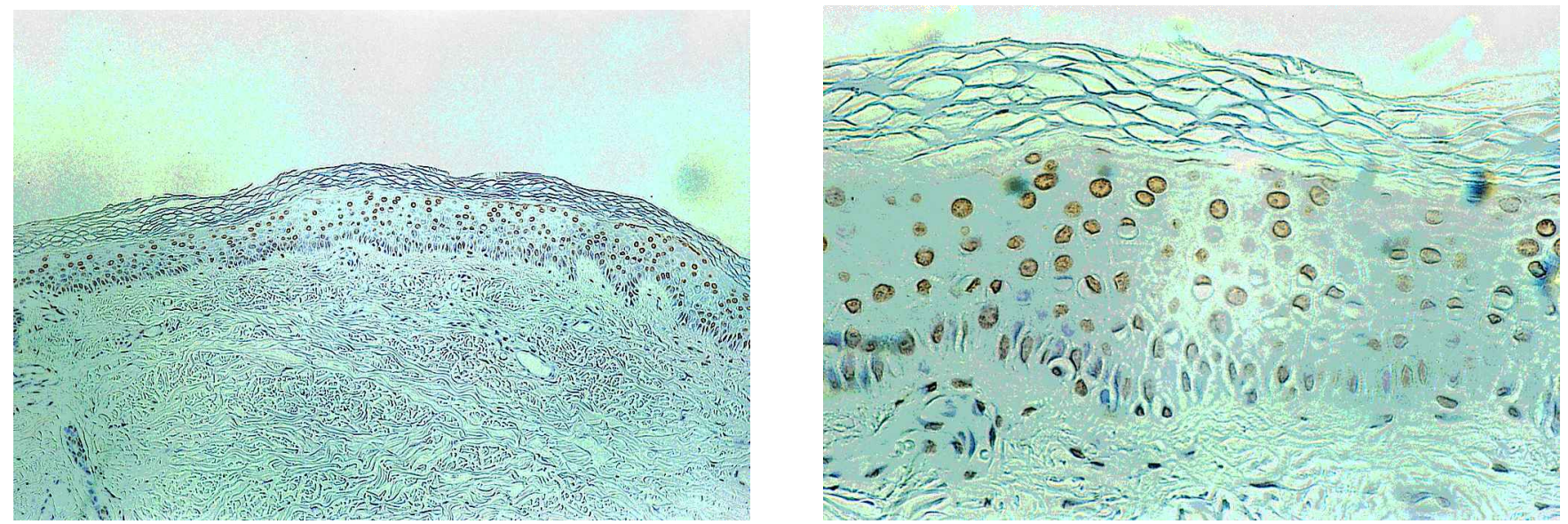

Fig. 3 Artifactual TUNEL positivity resulting from cutting of tissue sections. (A) Clinical GVHD specimen, showing scattered TUNEL-positive cells throughout tissue section. (B) Higher-power view of (A), showing lack of apoptotic morphology in TUNEL-positive cells.

tissue a reasonable distance $(>80 \mu \mathrm{m})$ from the border of tissue sections.

Finally, we have also noted nuclei that stain moderately by the TUNEL reaction and which are scattered relatively evenly throughout the specimen (Fig. 3A,B). These nuclei did not show morphology suggestive of apoptosis, but rather had normal cytological features. The intensity of
TUNEL staining of these cells was generally less than that of clearly apoptotic cells with apoptotic morphology. While it was possible that these moderately staining, morphologically normal cells actually represented cells in an early state of apoptosis, the large number of such cells made this explanation somewhat unlikely. We therefore examined the possibility that such TUNEL positivity could actually

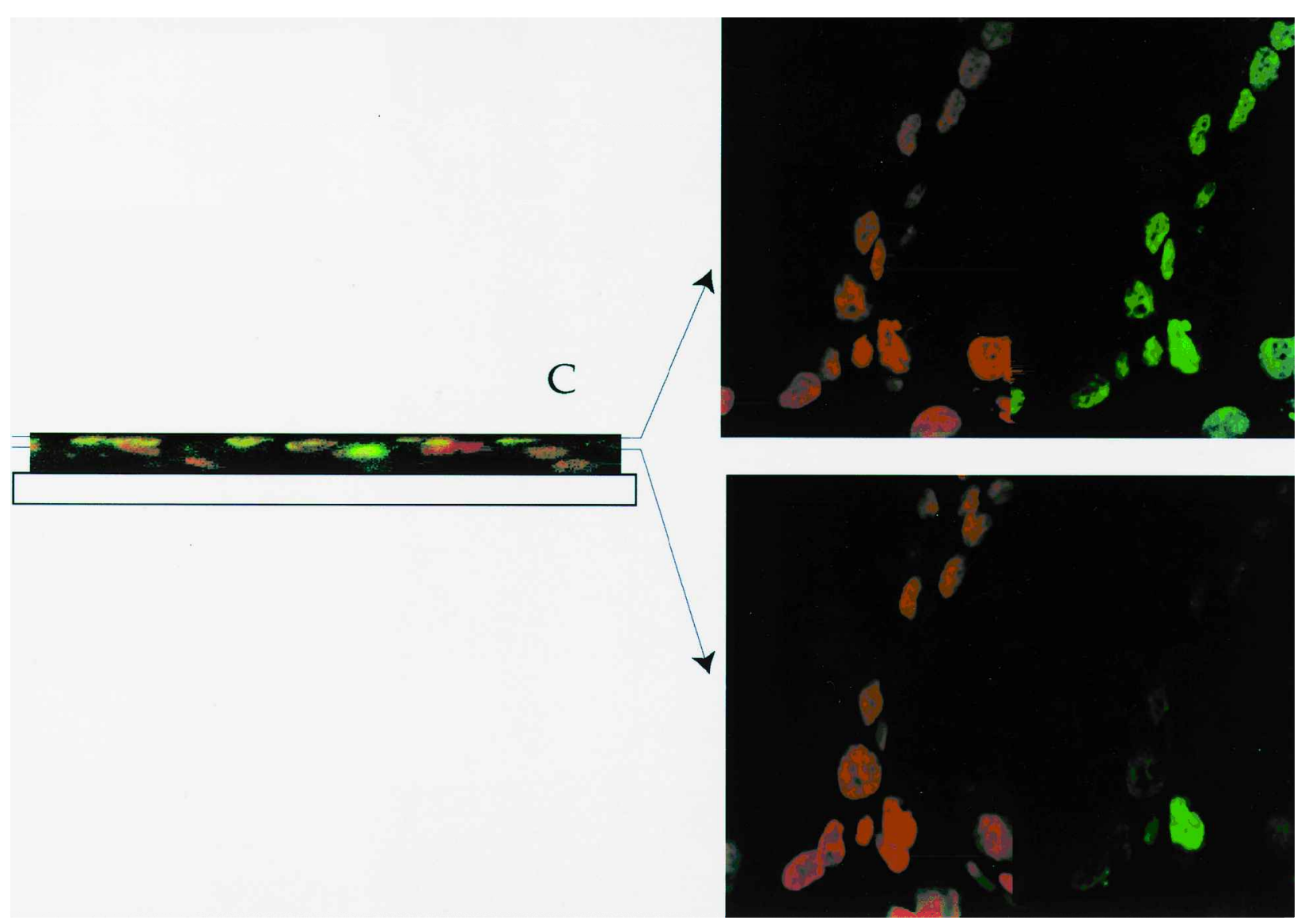

Fig. 4 LCSM image of 30- $\mu \mathrm{M}$ thick section of involuting mouse mammary gland stained for all nuclei by propidium iodide (red), and by TUNEL for apoptotic nuclei (green). In the focal plane near the cutting surface of the section (top right), all nuclei show TUNEL positivity. In the focal plane below the cutting surface (bottom right), only scattered cells show TUNEL positivity. Figure at left is a $z$-axis reconstruction created using IMARIS software (Zurich, Switzerland). 
represent detection of DNA strand breaks induced during cutting of tissue sections. During cutting of tissue, some cell nuclei within the specimen are transected by the cutting surface, and shear forces during the cutting process can disrupt DNA within the nucleus. Such disrupted DNA strands could in theory be detected by the TUNEL assay. To investigate this possibility, we performed laser confocal scanning microscopy (LCSM) on TUNEL-stained tissue sections. LCSM allows highly precise focusing on very narrow focal planes within the specimen, thus allowing us to evaluate the patterns of TUNEL positivity within various planes of a given tissue section. As can be seen in Fig. 4, in the areas of the section between the cutting surfaces, only scattered cells with apoptotic morphology show TUNEL positivity. In contrast, at the superficial cutting surface all severed nuclei show TUNEL positivity. Since the vast majority of these cells show normal morphology, and since there is no biologically plausible mechanism by which cells at the surface of a tissue section would be expected to preferentially undergo apoptosis, it is highly likely that the TUNEL positivity of these cells represents detection of DNA breaks occurring as a result of the section-cutting process. In contrast to the superficial cut surface, the deep cut surface adjacent to the microscope slide showed only a few TUNEL-positive cells. It is likely that the free DNA ends of these cut nuclei interact with the coating layer of the microscope slide, minimising the interaction of these artifactual free DNA ends with the terminal transferase. A similar artifact has recently been reported by Sloop et al., who described increased TUNEL positivity near the surface of thick $(15-\mu \mathrm{m})$ sections of stomach. ${ }^{12}$

In summary, the TUNEL assay has several potential pitfalls - the main being the lack of specificity for apoptotic cell death. Positive signals may be seen in non-apoptotic tissues for a number of reasons, ranging from cell death by non-apoptotic mechanisms to artifacts of tissue fixation or specimen cutting. In our laboratory, we consider cells as truly apoptotic only when TUNEL positivity is accompanied by clear apoptotic morphology. By these criteria, the TUNEL assay serves mainly as an aid to draw attention to cells with apoptotic morphology. Although apoptotic keratinocytes in skin biopsies can be identified by morphology without TUNEL staining, in our hands the TUNEL assay increased the number of apoptotic cells identified in a given tissue section by two- to three-fold in a blinded study. ${ }^{1}$ However, while use of the TUNEL assay may increase the sensitivity of detection of apoptotic keratinocytes, the clinical significance of this remains to be established. By accepting as true-positives only TUNEL-positive cells with apoptotic morphology, it is likely that we are missing cells in the earlier stages of apoptosis. It is possible to perform the TUNEL assay in such a way as to obtain a positive signal in these cells, but in our experience this leads to an unacceptable loss of specificity for apoptotic cell death. Furthermore, there are clearly even earlier stages of apoptotic death in which the cells have irreversibly activated their death programmes, but have not yet begun the process of DNA fragmentation. ${ }^{13}$ Future advances in the identification of apoptotic cell death in tissue sections may highlight such cells in the early stages of death, but the clinical utility of identifying such cells in GVHD or other processes will remain an outstanding question until that time.

Address for correspondence: K.R.J., MD, PhD, UW Virology Lab at FHCRC, D3-100, 1100 Fairview Ave. N, Seattle, WA 98109, USA.

\section{References}

1. Jerome KR, Conyers SJ, Hansen DA, Zebala JA. Keratinocyte apoptosis following bone marrow transplantation: evidence for CJLdependent and -independent pathways. Bone Marrow Transpl 1998; 22: 359-66.

2. Bomb'i JA, Nadal A, Carreras E, et al. Assessment of histopathological changes in the colonic biopsy in acute graft-versus-host disease. Am J Clin Pathol 1995; 103: 690-5.

3. Ferrara J, Guillen FJ, Sleckman B, et al. Cutaneous acute graft-versushost disease to minor histocompatibility antigens in a murine model: histological analysis and correlation to clinical disease. J Invest Dermatol 1986; 86: 371-5.

4. Langley RG, Walsh N, Nevill T, et al. Apoptosis is the mode of keratinocyte death in cutaneous graft-versus-host disease. J Am Acad Dermatol 1996; 35: 187-90.

5. Gilliam AC, Whitaker-Menezes D, Korngold R, Murphy GF. Apoptosis is the predominant form of epithelial target cell injury in experimental graft-versus-host disease. J Invest Dermatl 1996; 107: 377-83.

6. Gavrieli Y, Sherman Y, Ben-Sasson SA. Identification of programmed cell death in situ via specific labeling of nuclear DNA fragmentation. J Cell Biol 1992: 119: 493-501.

7. Yasuda M, Umemura S, Osamura RY, et al. Apoptotic cells in the human endometrium and placental villi: pitfalls in applying the TUNEL method. Arch Histol Cytol 1995; 58: 185-90.

8. Grasl-Kraupp B, Ruttkay-Nedecky B, Koudelka H, et al. In situ detection of fragmented DNA (TUNEL assay) fails to discriminate among apoptosis, necrosis, and autolytic cell death: a cautionary note. Hepatology 1995; 21: 1465-8.

9. Gold R, Schmied M, Giegerich G, et al. Differentiation between cellular apoptosis and necrosis by the combined use of in situ tailing and nick translation techniques. Lab Invest 1994; 71: 219-25.

10. Charriaut-Marlangue C, Ben-Ari Y. A cautionary note on the use of the TUNEL stain to determine apoptosis. NeuroReport 1995; 7: 61-4.

11. Tateyama $\mathrm{H}$, Tada $\mathrm{T}$, Hattori $\mathrm{H}$, et al. Effects of prefixation and fixation times on apoptosis detection by in situ end-labeling of fragmented DNA. Arch Pathol Lab Med 1998; 122: 252-5.

12. Sloop GD, Roa IC, Delgado AG, et al. Histologic sectioning produces TUNEL reactivity. A potential cause of false-positive staining. Arch Pathol Lab Med 199; 123: 529-32.

13. Cohen GM, Sun X-M, Snowden RT, et al. Key morphological features of apoptosis may occur in the absence of internucleosomal DNA fragmentation. Biochem J 1992; 286: 331-4. 